\title{
Hypotonia, Ataxia, and Delayed Development Syndrome caused by the EBF3 mutation in a Korean boy with muscle hypotonia
}

\author{
Tae-Gyeong Kim ${ }^{1 \oplus}$, Yoon-Ha Choi ${ }^{1 \oplus}$, Ye-Na Lee ${ }^{1 \oplus}$, Min-Ji Kang ${ }^{2 \oplus}$, Go Hun Seo ${ }^{3 \oplus}$, and Beom Hee Lee ${ }^{1,2 * \odot}$ \\ ${ }^{1}$ Department of Pediatrics, Asan Medical Center, University of Ulsan College of Medicine, Seoul, Korea \\ ${ }^{2}$ Genome Research Center for Birth Defects and Genetic Diseases, Asan Medical Center, University of Ulsan College of Medicine, Seoul, Korea \\ ${ }^{3} 3$ Billion, Inc., Seoul, Korea
}

\begin{abstract}
Hypotonia, Ataxia, and Delayed Development Syndrome (HADDS) is an autosomal-dominant, extremely rare neurodevelopmental disorder caused by the heterozygous EBF3 gene mutation. EBF3 is located on chromosome 10q26.3 and acts as a transcription factor that regulates neurogenesis and differentiation. This syndrome is characterized by dysmorphism, cerebellar hypoplasia, urogenital anomaly, hypotonia, ataxia, intellectual deficit, and speech delay. The current report describes a 3-year-old Korean male carrying a de novo EBF3 mutation, c.589A>G (p.Asn197Asp), which was identified by whole exome sequencing. He manifested facial dysmorphism, hypotonia, strabismus, vermis hypoplasia, and urogenital anomalies, including vesicoureteral reflux, cryptorchidism, and areflexic bladder. This is the first report of a case of HADDS cause by an EBF3 mutation in the Korean population.
\end{abstract}

Key words: Hypotonia, Ataxia, and Delayed Development Syndrome, EBF3, Muscle hypotonia, Developmental disabilities.

\section{Introduction}

Hypotonia, Ataxia, and Delayed Development Syndrome (HADDS; OMIM 617330) is an extremely rare human neurodevelopmental syndrome caused by a heterozygous loss-of-function mutation in the EBF3 gene on chromosome 10q26. Patients show congenital hypotonia, structural brain malformations, ataxia, and genitourinary abnormalities [1]. HADDS was first described in 2016. Its incidence rate is unknown; however, 33 patients have been reported to carry a de novo EBF3 mutation to date, which has been identified by whole exome sequencing (WES) [1-5].
Here, we describe a 3-year-old child who presented with dysmorphism, developmental delay, vesicoureteral reflux (VUR), strabismus, and vermian hypoplasia. WES identified a de novo heterozygous EBF3 mutation, c.589A $>G$ (p.Asn197Asp). This is the first case of HADDS reported in Korean population.

\section{Case}

A 2-month-old male was referred to Medical Genetics Center, Asan Medical Center, Seoul, Korea due to hypotonia. He was born after 39 weeks of gestation to a 24-year-old mother, who had previously experienced 2 spontaneous abortions. Family

Received: 4 September 2020, Revised: 6 October 2020, Accepted: 7 October 2020, Published: 31 December 2020

${ }^{*}$ Corresponding author: Beom Hee Lee, M.D., Ph.D. (iD https://orcid.org/0000-0001-9709-2631

Department of Pediatrics, Asan Medical Center, University of Ulsan College of Medicine, 88 Olympic-ro 43-gil, Songpa-gu, Seoul 05505, Korea.

Tel: +82-2-3010-5950, Fax: +82-2-473-3725, E-mail: bhlee@amc.seoul.kr

Conflict of interest: The authors declare that they do not have any conflicts of interest.

(ㄷ) This is an open-access article distributed under the terms of the Creative Commons Attribution Non-Commercial License (http://creativecommons.org/licenses/by-nc/4.0/) which permits unrestricted non-commercial use, distribution, and reproduction in any medium, provided the original work is properly cited.

(c) Copyright 2020 by the Korean Society of Medical Genetics and Genomics 
history for developmental delay was rejected. His birth weight was $2.95 \mathrm{~kg}$ (10-50th percentile), height was $47.6 \mathrm{~cm}$ (10th percentile), and head circumference was $34 \mathrm{~cm}$ (10-50th percentile). He was admitted to the neonatal intensive care unit at birth due to perinatal distress. Dysmorphic features were reported at admission, such as a long and triangular face, hypertelorism, low set ears, high nasal bridge, broad nasal tip, short anteverted nostrils, deep philtrum, and downturned corners of the mouth. In addition, short stature, hypotonia, constipation, micropenis, cryptorchidism, VUR, and clubfoot were noted.

At 3 months of age, a left VUR grade $V$ with ureterovesical stenosis was observed following urinary tract infection (Fig. 1). At 6 months of age, he was unable to control his head. In addition, his muscle tone was decreased, with a normoactive deep tendon reflex. Brain magnetic resonance imaging (MRI) showed prominent retrocerebellar CSF spaces with suspected vermian hypoplasia (Fig. 2). His karyotype is 46, XY; chromosomal microarray did not find any significant copy number variant.

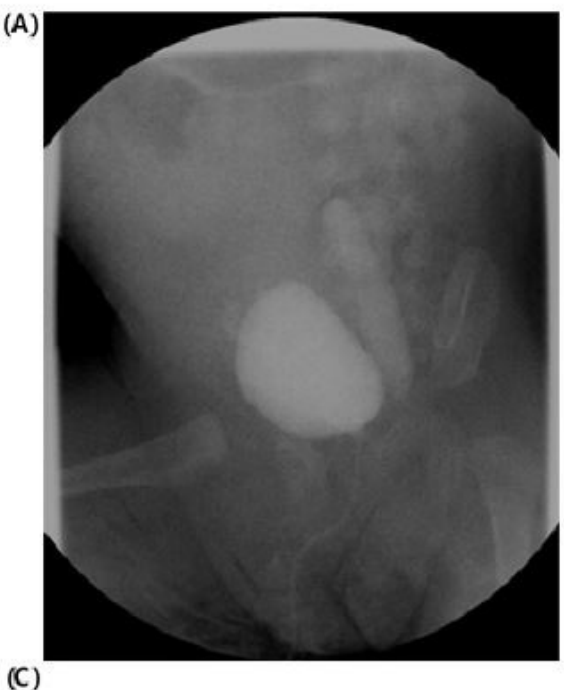

(B)

\begin{tabular}{llll}
$\begin{array}{l}\text { Table of Resut Summary } \\
\text { Parameters }\end{array}$ & Left & Rught & Total \\
\hline Split Function (\%) & 42.8 & 57.2 & \\
Kidnev Counts (cpm) & 5910.1 & 7903.9 & 13814 \\
Time of Max (min) & 19.5 & 19.5 & \\
Time of 'YMax (min) & & &
\end{tabular}

\section{Renal(Mag3) [Results] 3/27/2017

$$
\text { 1. }
$$

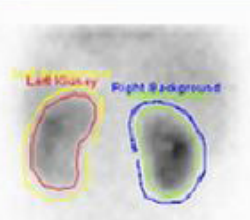

(C)

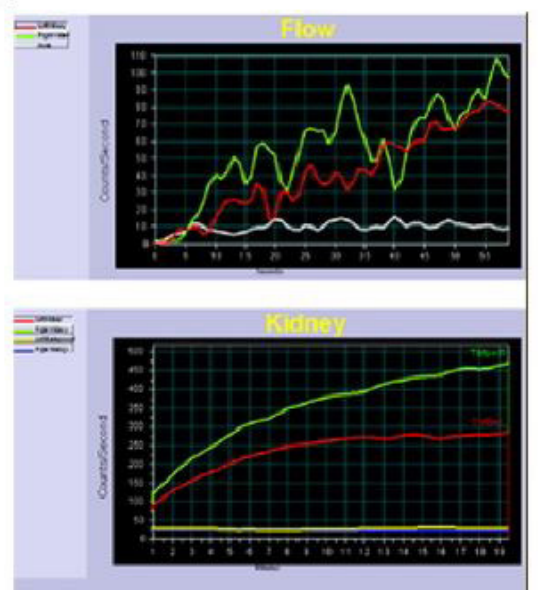

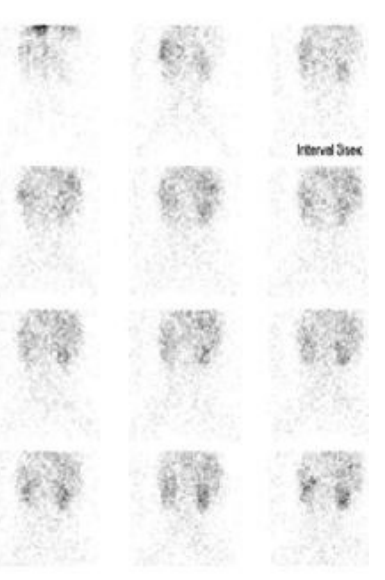

Fig. 1. (A) Voiding cystourethrogram showed left vesicoureteral reflux grade $V$ at 3 months of age. (B) Tc-99m MAG3 renal scan showed decreased perfusion of left kidney. (C) The cortical transit time of the left kidney is delayed compared with the right kidney. Pelvicalyceal retention is present in both kidneys, which means delayed excretion of the left kidney.

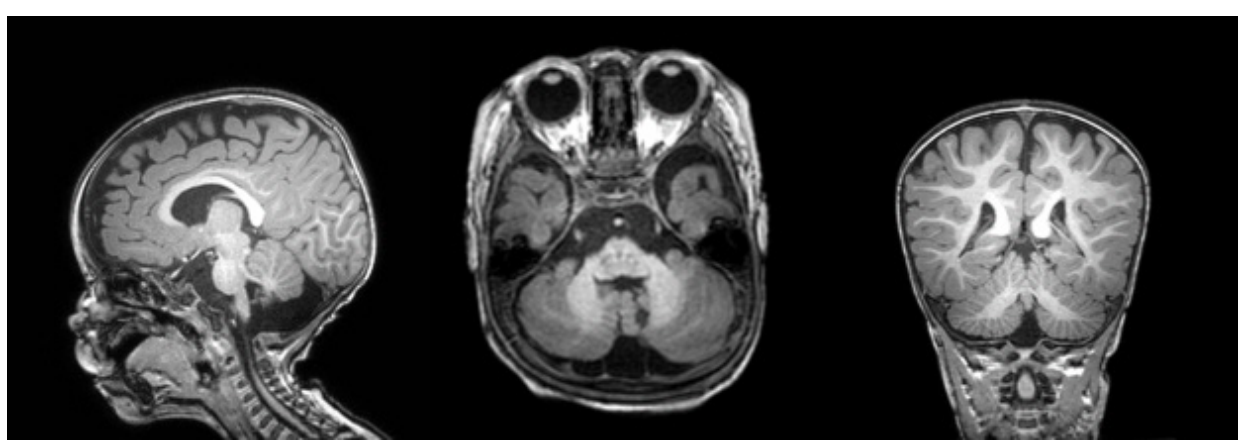

Fig. 2. Brain magnetic resonance imaging of the patient at 1 year of age. Extra-axial, retrocerebellar cerebrospinal fluid space was prominent with suspicious vermian hypoplasia. 
At 9 months of age, ureteroneocystostomy was performed for left obstructive refluxing megaureter and bilateral orchiopexy for cryptorchidism. Strabismus, ataxic hand movement, and insensitivity to pain were noted. At that time, the patient's height was $65 \mathrm{~cm}$ (<3rd percentile), his head circumference was 44.5 $\mathrm{cm}$ (10-50th percentile), and body weight was $6.9 \mathrm{~kg}$ (3th per-

I

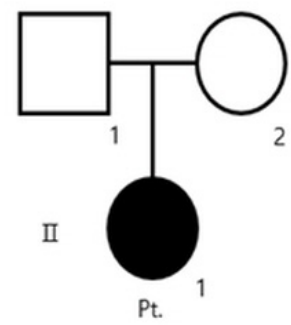

I:1

I:2

II:1

$E B F 3$, c.589A $>\mathrm{G}$ (p.Asn197Asp)

vUS
G T T C T GT TCT

G T T C T

\section{GT T C I}
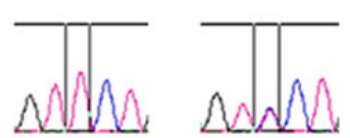
G T C C T

Fig. 3. The novel missense variant, c.589A $>6$ (p.Asn197Asp), in the $E B F 3$ gene of the patient (Il:1). VUS, variants of uncertain significance.

centile).

The Denver Development Scale Test (DDST) at 12 months revealed global developmental delay; his developmental stages were 6, 6-7, 2-3, and 5-6 months in each personal-social, fine motor-adaptive, language, and gross motor domain, respectively.

Based on the multi-systemic clinical features, including global developmental delay, short stature, brain anomaly, genitourinary abnormality, and normal chromosomal microarray results, WES was performed, as described previously. Informed consent was obtained from the parents. This study was approved by the Institutional Review Board for Human Research of Asan Medical Center (IRB number 2017-0988).

WES identified a novel mutation, c.589A $>$ G (p.Asn197Asp), in the EBF3 gene (Fig. 3). In silico analysis predicted that p.Asn 197Asp would alter protein function (MutationTaster; Polyphen-2 and SIFT), which has not been reported in a general population cohort (https://gnomad.broadinstitute.org/). This variant was classified as "uncertain significance", according to the American College of Medical Genetics and Genomics guidelines [6]. His parents did not carry the mutation. This led to diagnostic confirmation of HADDS.

Echocardiography revealed a retroaortic left innominate vein with hemiazygos inflow and no other structural cardiac anomaly. Anticholinergic and laxative drugs were prescribed for

Table 1. Clinical manifestations of Hypotonia, Ataxia, and Delayed Development Syndrome patients

\begin{tabular}{|c|c|c|c|c|c|c|c|}
\hline Clinical features & Harms $(n=10)$ & Chao $(n=3)$ & Sleven $(n=8)$ & Tanaka $(\mathrm{n}=7)$ & Blackburn $(n=5)$ & Total number & Our case \\
\hline Hypotonia & $10 / 10$ & $3 / 3$ & $8 / 8$ & $6 / 7$ & $5 / 5$ & $32 / 33(96.9)$ & + \\
\hline Ataxia issue & $6 / 10$ & $1 / 3$ & $8 / 8$ & $5 / 7$ & $4 / 5$ & $24 / 33(72.7)$ & + \\
\hline Intellectual disability & $10 / 10$ & $3 / 3$ & $8 / 8$ & - & $5 / 5$ & 26/26 (100.0) & + \\
\hline Speech delay & $10 / 10$ & $3 / 3$ & $8 / 8$ & $7 / 7$ & $5 / 5$ & $33 / 33(100.0)$ & + \\
\hline High pain tolerance & - & $2 / 3$ & 1 & 2 & 2 & 7 & + \\
\hline Facial dysmorphism & $6 / 8$ & $3 / 3$ & $7 / 8$ & - & $5 / 5$ & 21/24 (87.5) & + \\
\hline Autistic feature $^{\mathrm{a}}$ & 2 & 1 & 3 & $6 / 7$ & - & 12 & Unevaluable \\
\hline Brain MR abnormality & $2 / 8$ & $2 / 3$ & $3 / 8$ & $3 / 6$ & $3 / 5$ & $13 / 30(43.3)$ & + \\
\hline Vermis hypoplasia & $2 / 8$ & $2 / 3$ & $1 / 8$ & $1 / 6$ & $3 / 5$ & 9/30 (30.0) & + \\
\hline Seizure & $2 / 9$ & - & $3 / 8$ & $0 / 7$ & $0 / 5$ & $5 / 29(17.2)$ & - \\
\hline Strabismus & $9 / 10$ & $2 / 3$ & $6 / 8$ & $4 / 6$ & $5 / 5$ & 26/32 (81.3) & + \\
\hline Genitourinary anomaly & 3 & $2 / 3$ & 4 & $5 / 7$ & $5 / 5$ & 19 & + \\
\hline VUR & 1 & - & 3 & 1 & 2 & 7 & + \\
\hline Bladder dysfunction & 1 & - & 1 & 1 & 2 & 5 & + \\
\hline Congenital heart defect ${ }^{c}$ & 1 & - & - & - & - & 1 & + \\
\hline Constipation & 2 & - & - & 1 & - & 3 & + \\
\hline
\end{tabular}

Values are presented as number only or number (\%).

MR, magnetic resonance; VUR, vesicoureteral reflux.

${ }^{a}$ Including attention deficit, and limited facial expression. ${ }^{\mathrm{b}}$ Including cryptorchidism, hypospadias, dysplastic kidney, VUR. Reduction in volume of the labia majora, micropenis, and recurrent urinary tract infection. 'Atrial septal defect. 
areflexic bladder and constipation, respectively.

At the latest evaluation at 2 years and 10 months of age, developmental status was severely delayed despite comprehensive rehabilitation treatment. DDST scores were 7,6, 6, and 9 months in each personal-social, fine motor-adaptive, language, and gross motor domain, respectively.

\section{Discussion}

In the current report, we described the first Korean case of HADDS caused by an EBF3 mutation, which was identified by WES. Our case shares the clinical manifestations of previously reports describing HADDS. Our patient showed the characteristic facial dysmorphism, including long and triangular face, hypertelorism, low set ears, high nasal bridge, broad nasal tip, short anteverted nostrils, deep philtrum, and downturned corners of the mouth. The most common symptoms are hypotonia and developmental delay, especially speech delay. Additional features, such as strabismus, genitourinary abnormalities, high pain tolerance, and autistic features, including lack of eye contact, repetitive activities, and impaired affective expression, have been reported. Brain MRI features include vermian hypoplasia, cerebellar atrophy, cerebellar arachnoid cyst, and delayed myelination (Table 1) [1-5]. In addition, previous studies by Lin et al. [7] have reported that patients with a 10q26 deletion, including EBF3 haploinsufficiency, show the overlapping features, such as a prominent or beaked nose, broad nasal bridge, hypertelorism, webbed neck, scoliosis, micropenis, cryptorchidism, kidney abnormalities, attention-deficit/hyperactivity disorder, and impulsivity.

EBF3 is located on chromosome 10q26.3; it encodes a member of the collier/olfactory-1/EBF (COE) family of transcription factors that regulate neurogenesis and differentiation [8]. EBF3 is expressed in Cajal-Retzius (CR) cells, which are important for the development of cerebral cortex. Chiara et al. [9] reported that this protein controls the migration of CR cells arising in the brain cortex. The pathogenic mechanism of EBF3 mutations is loss-of-function, which results in reduced EBF3 activity and subsequent aberration of neurodevelopment [4]. Moreover, EBF3 is expressed in the urinary system, reproductive system, and metanephros [10]; therefore, its deficiency is associated with urogenital abnormalities.

To date, 27 out of 33 patients who have been diagnosed with HADDS with a proven EBF3 mutation exhibit a mutation in COE1 DNA binding domain. In this domain, 11 patients showed a mutation in the zinc finger motif, which is crucial for zinc coordination and positioning residues for DNA binding. These mutations are expected to decrease the affinity of mutant EBF3 to DNA, resulting in aberrant DNA binding [2]. Interestingly, only one patient has been reported to carry an EBF3 mutation in the transactivation domain mutation [5]. Two patients were identified with a mutation in the lg-like, plexins, transcription factor and helix-loop-helix domains [3]. It is possible that aberrant EBF3-mediated transcription in the muscular systems could lead to hypotonia, atonic bladder, loss of bladder control, and other phenotypes observed in patients. The novel missense mutation of our patient, p.Asn 197Asp, is located in the DNA binding domain [11].

In conclusion, this is the first report of a case with HADDS in the Korean population caused by a de novo EBF3 mutation, c.589A $>G$ (p.Asn 197Asp). The identification of more cases will help to understand this extremely rare genetic disease, which will improve patient care.

\section{Acknowledgements}

This research was supported by Institute for Information and Communications Technology Promotion (IITP) grant funded by the Korean government (MSIT) (2018-0-00861, Intelligent SW Technology Development for Medical Data Analysis).

\section{References}

1. Chao HT, Davids M, Burke E, Pappas JG, Rosenfeld JA, McCarty AJ, et al. A syndromic neurodevelopmental disorder caused by de novo variants in EBF3. Am J Hum Genet 2017;100:128-37.

2. Blackburn PR, Barnett SS, Zimmermann MT, Cousin MA, Kaiwar C, Pinto $E$ Vairo $F$, et al. Novel de novo variant in EBF3 is likely to impact DNA binding in a patient with a neurodevelopmental disorder and expanded phenotypes: patient report, in silico functional assessment, and review of published cases. Cold Spring Harb Mol Case Stud 2017;3:a001743.

3. Harms FL, Girisha KM, Hardigan AA, Kortüm F, Shukla A, Alawi M, et al. Mutations in EBF3 disturb transcriptional profiles and cause intellectual disability, ataxia, and facial dysmorphism. Am J Hum Genet 2017;100:117-27.

4. Sleven H, Welsh SJ, Yu J, Churchill MEA, Wright CF, Henderson A, et al. De novo mutations in EBF3 cause a neurodevelopmental syndrome. Am J Hum Genet 2017;100:138-50.

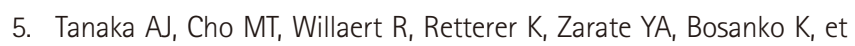
al. De novo variants in EBF3 are associated with hypotonia, developmental delay, intellectual disability, and autism. Cold Spring Harb Mol 
Case Stud 2017;3:a002097.

6. Richards S, Aziz N, Bale S, Bick D, Das S, Gastier-Foster J, et al.; ACMG Laboratory Quality Assurance Committee. Standards and guidelines for the interpretation of sequence variants: a joint consensus recommendation of the American College of Medical Genetics and Genomics and the Association for Molecular Pathology. Genet Med 2015;17:405-24.

7. Lin S, Zhou Y, Fang Q, Wu J, Zhang Z, Ji Y, et al. Chromosome $10 q 26$ deletion syndrome: two new cases and a review of the literature. Mol Med Rep 2016;14:5134-40.

8. Wang SS, Lewcock JW, Feinstein P, Mombaerts P, Reed RR. Genetic disruptions of O/E2 and O/E3 genes reveal involvement in olfactory receptor neuron projection. Development 2004;131:1377-88.
9. Chiara F, Badaloni A, Croci L, Yeh ML, Cariboni A, Hoerder-Suabedissen $A$, et al. Early B-cell factors 2 and 3 (EBF2/3) regulate early migration of Cajal-Retzius cells from the cortical hem. Dev Biol 2012;365:27789

10. Harding SD, Armit $C$, Armstrong J, Brennan J, Cheng $Y$, Haggarty $B$, et al. The GUDMAP database--an online resource for genitourinary research. Development 2011;138:2845-53.

11. Siponen MI, Wisniewska M, Lehtiö L, Johansson I, Svensson L, Raszewski $G$, et al. Structural determination of functional domains in early B-cell factor (EBF) family of transcription factors reveals similarities to Rel DNA-binding proteins and a novel dimerization motif. J Biol Chem 2010;285:25875-9. 